\title{
Transcriptional regulation of the thyrotropin-releasing hormone gene by leptin and melanocortin signaling
}

\author{
Mark Harris, ${ }^{1}$ Carl Aschkenasi, ${ }^{1}$ Carol F. Elias, ${ }^{1}$ Annie Chandrankunnel, ${ }^{1}$ \\ Eduardo A. Nillni, ${ }^{2}$ Christian Bjørbæk, ${ }^{1}$ Joel K. Elmquist, ${ }^{1,3}$ Jeffrey S. Flier, ${ }^{1}$ \\ and Anthony N. Hollenberg ${ }^{1}$
}

${ }^{1}$ Division of Endocrinology, Beth Israel Deaconess Medical Center and Harvard Medical School, Boston, Massachusetts, USA

${ }^{2}$ Division of Endocrinology, Rhode Island Hospital and Brown University School of Medicine, Providence, Rhode Island, USA

${ }^{3}$ Department of Neurology, Beth Israel Deaconess Medical Center and Program in Neuroscience, Harvard Medical School,

Boston, Massachusetts, USA

Address correspondence to: Anthony N. Hollenberg, Division of Endocrinology, Beth Israel Deaconess Medical Center-

Research North, 330 Brookline Avenue, Boston, Massachusetts 02215, USA.

Phone: (617) 667-2151; Fax: (617) 667-2927; E-mail: thollenb@caregroup.harvard.edu.

Received for publication July 11, 2000, and accepted in revised form November 21, 2000.

\begin{abstract}
Starvation causes a rapid reduction in thyroid hormone levels in rodents. This adaptive response is caused by a reduction in thyrotropin-releasing hormone (TRH) expression that can be reversed by the administration of leptin. Here we examined hypothalamic signaling pathways engaged by leptin to upregulate TRH gene expression. As assessed by leptin-induced expression of suppressor of cytokine signaling-3 (SOCS-3) in fasted rats, TRH neurons in the paraventricular nucleus are activated directly by leptin. To a greater degree, they also contain melanocortin-4 receptors (MC4Rs), implying that leptin can act directly or indirectly by increasing the production of the MC4R ligand, $\alpha$-melanocyte stimulating hormone ( $\alpha$-MSH), to regulate TRH expression. We further demonstrate that both pathways converge on the TRH promoter. The melanocortin system activates the TRH promoter through the phosphorylation and DNA binding of the cAMP response element binding protein (CREB), and leptin signaling directly regulates the TRH promoter through the phosphorylation of signal transducer and activator of transcription 3 (Stat3). Indeed, a novel Stat-response element in the TRH promoter is necessary for leptin's effect. Thus, the TRH promoter is an ideal target for further characterizing the integration of transcriptional pathways through which leptin acts.
\end{abstract}

J. Clin. Invest. 107:111-120 (2001).

\section{Introduction}

The hypothalamic peptide, thyrotropin-releasing hormone (TRH), is essential for the normal production of thyroid-stimulating hormone in the pituitary and thyroid hormones in the thyroid gland (1). Within the paraventricular hypothalamic nucleus (PVH), TRH is regulated at the transcriptional level by thyroid hormone (T3), such that in hyperthyroid states TRH expression is reduced and in hypothyroid states its expression is increased (2). Recently, it has become clear that TRH is also regulated by nutritional states. To conserve energy during periods of food deprivation, rodents dramatically reduce their thyroid hormone levels, which in turn allows reductions in their metabolic rate (3). This adaptation to starvation is accomplished through a reduction in the expression of TRH in the PVH, indicating that a central process contributes to the regulation of this physiological adaptation. The mechanism governing the nutritional regulation of TRH has been clarified by Ahima et al., who reversed starvation-induced suppression of thyroid hormone levels in mice by administering leptin during starvation (4). This finding was extended by Legradi et al., who further demonstrated that leptin's effects were due to upregulation of TRH gene expression (5).
Although leptin can regulate TRH gene expression in the $\mathrm{PVH}$, it remains unclear whether this effect is mediated by direct actions of leptin on the TRH neuron in the PVH or indirectly through leptin effects on other neurons, which then project to the TRH neuron. Recently Legradi et al. demonstrated that chemical ablation of the arcuate nucleus blocks leptin's effect on TRH expression, suggesting that an indirect pathway is involved (6). Given that leptin activates pro-opiomelanocortin (POMC) gene expression in the arcuate nucleus, POMC-derived $\alpha$-melanocyte stimulating hormone $(\alpha-\mathrm{MSH})$ is a candidate hormone that may be responsible for the regulation of TRH expression $(7,8)$. $\alpha$-MSH signals through the melanocortin 4 receptor (MC4R), which is responsible for many of the central effects of melanocortin signaling. The role of $\alpha-\mathrm{MSH}$ in regulating the TRH neuron has now been documented in two recent reports. Fekete et al. have demonstrated that $\alpha$-MSH neurons innervate the TRH-neuron in the PVH and that centrally administered $\alpha-\mathrm{MSH}$ prevents the drop in TRH expression induced by starvation and partially rescues the drop in thyroid hormone levels. (9). Kim et al. demonstrated that $\alpha-\mathrm{MSH}$ increases TSH levels when it is given centrally and that 
it stimulates TRH release in hypothalamic slices (10). Moreover, the MC4R antagonist AgRP (11) inhibits the effects of leptin on TRH release from hypothalamic slices. Taken together, these data suggest that engagement of the melanocortin pathway is required for leptin to regulate TRH expression. However, recent work by Nillni et al. has shown that TRH neurons coexpress the leptin receptor in primary cultures of fetal rat hypothalamic neurons. In addition, these neurons synthesize and secrete TRH protein in response to leptin (12). Also, previous work has demonstrated the existence of the long form of the leptin receptor (ObRb) in the PVH and that leptin can induce c-Fos expression in the $\mathrm{PVH}$ $(13,14)$. Thus, potential direct effects of leptin on the TRH neuron need to be explored further.

Regulation of the TRH gene is not well understood. Work from a number of groups including our own has established that the murine, rat, and human TRH promoters all contain a critical thyroid hormone receptor binding site, termed Site 4, in the proximal promoter $(15,16)$. This site appears to be critical for binding of the thyroid hormone receptor (TR) isoforms and necessary for negative regulation by T3. Interestingly, Site 4 also acts as a strong basal enhancer of the promoter in a number of cell lines and bears a strong resemblance to a cAMP response element. However, its role in vivo has not heretofore been studied. Because little information exists about systems that upregulate TRH gene expression, very little is known about other transcriptionally important pathways that target the TRH promoter.

In this study, we have further defined the signaling systems involved in the regulation of TRH gene expression. Through in situ hybridization for MC4Rs and SOCS-3, we demonstrate that the TRH neuron in the $\mathrm{PVH}$ can be acted on directly by both the melanocortin and leptin signaling systems. In addition, we demonstrate that the TRH promoter responds to both signaling pathways through specific cis-acting sequences that bind the transcription factors cAMP response element binding protein (CREB) and signal transducers and activators of transcription-3 (Stat3). Indeed, CREB and Stat 3 play an important role in the regulation of TRH gene expression by $\alpha-\mathrm{MSH}$ and leptin, respectively. Furthermore, the T3 signaling pathway overlaps with the leptin and melanocortin pathways, suggesting that the set point of TRH gene expression is determined by multiple inputs.

\section{Methods}

Animals. Adult male pathogen-free Sprague-Dawley rats were housed in a light and temperature controlled environment with water and standard chow available ad libitum. The animals and procedures used were approved by the Harvard Medical School and Beth Israel Deaconess Medical Center Institutional Animal Care and Use Committees. To examine for MC4R expression, adult male pathogen-free Sprague-Dawley rats were used $(n=3)$. To enable leptin treatment, intravenous femoral vein catheters were surgically implanted (17). After a 1-week recovery period, the animals were fasted for 48 hours and subsequently administered pyrogen-free saline $(n=3$; Sigma Chemical Co., St. Louis, Missouri, USA) or recombinant murine leptin $(n=5 ; 1.0 \mathrm{mg} / \mathrm{kg}$; kindly provided by Eli Lilly, Indianapolis, Indiana, USA) via the catheter. The animals were then sacrificed 1 hour after administration of leptin or saline.

In situ bybridization histochemistry. All animals were sacrificed by intracardiac perfusion of $10 \%$ neutral buffered formalin and postfixed in this solution for 3-4 hours at room temperature. Brains of perfused animals were excised and dehydrated in $20 \%$ sucrose for approximately 48 hours, and frozen coronal sections then cut. Sections were mounted onto SuperFrost slides (Fisher, Pittsburgh, Pennsylvania, USA), air-dried, and stored at $-20^{\circ} \mathrm{C}$ with desiccant until further processing. Before hybridization, sections were subjected to a standard microwave procedure to expose hybridization substrates (18). Sections were then dehydrated again in ethanol, air-dried, and stored at $-20^{\circ} \mathrm{C}$ with desiccant.

The cDNA used for the generation of the SOCS-3 riboprobe has been described previously (19). The rat TRH and MC4R cDNAs used to generate riboprobes were created by RT-PCR from rat hypothalamic mRNA (primers available upon request). Generation of ${ }^{35} \mathrm{~S}$ and digoxigenin labeled riboprobes was performed as described previously using T7 polymerase (TRH) or SP6 polymerase (MC4R and SOCS-3) $(17,19,20)$. Hybridization solution containing riboprobes was applied to slides and incubated overnight at $56^{\circ} \mathrm{C}$. The next day, hybridized tissue was exposed to RNAse A for 30 minutes at $37^{\circ} \mathrm{C}$ and subsequently washed in decreasing solutions of saline sodium citrate buffer containing $0.25 \%$ dithiothreitol at $50^{\circ} \mathrm{C}, 55^{\circ} \mathrm{C}$, and $60^{\circ} \mathrm{C}$ for 1 hour each.

Sections were next incubated overnight in sheep antidigoxigenin antiserum conjugated to alkaline phosphatase (1:1,000), followed by overnight incubation in a nitroblue tetrazolium chloride and 5-bromo-4chloro-3-indolyl-phosphate chromagen solution (21). Sections were dipped in 3\% parlodion in isoamyl acetate, dried, and then dipped in NTB2 photographic emulsion (Eastman Kodak Co. Scientific Imaging Systems (New Haven, Connecticut, USA), stored at $4^{\circ} \mathrm{C}$ for 2-4 weeks and developed with D-19 developer (Eastman Kodak Co. Scientific Imaging Systems).

To estimate the percent of nonisotopically labeled TRH cells that were double labeled, background values of silver grain numbers were established in regions of the brain that did not exhibit specific MC4R or SOCS3 hybridization (cortex, amygdala, hippocampus, and caudoputamen) from each animal studied. The area counted was similar to the diameter of a digoxigeninlabeled PVH neuron. The relative number of silver grains overlying individual TRH neurons in the medial parvocellular divisions of the PVH were determined. A double-labeled cell was defined as having over threefold more grains versus the background from that derived from that animal studied. 
Plasmids and mammalian cell transfection. All human TRH promoter plasmids were derived from the -900 to +55 fragment cloned into $\mathrm{pA}_{3} \mathrm{LUC}$, which has been described previously (15). All further constructs besides the full-length promoter were truncated at +5 (SmaI site). The $-150,-50$, and $-150 \mathrm{M} 4$ constructs have been described previously (15). All other constructs were derived from the -150 series using PCR or restriction endonuclease digestion. The murine TRH promoter consists of a SmaI-SalI fragment encompassing from -255 to +12 of the murine promoter (a gift of M. Yamada). All TRH promoter constructs were cloned into $\mathrm{pA}_{3} \mathrm{LUC}$. RSV 180 contains the first $180 \mathrm{bp}$ of the rous sarcoma virus LTR and was also cloned into $\mathrm{pA}_{3} \mathrm{LUC}$ and has been described previously (22). GAS-Luc consists of five copies of the IFN- $\gamma$ Stat responsive element cloned upstream of the luciferase reporter (a gift of L. Stancato and R. Pine). The Flag-Stat murine expression vectors and murine ObRb expression vector have been described previously (23). The MC4R expression vector was also cloned into pcDNAI. The TR expression vectors contain the human cDNAs of the $\beta$ isoforms in $\mathrm{PKCR}_{2}$ and have been described previously (24). The wild-type and CREB mutant (S133A) were also cloned into $\mathrm{PKCR}_{2}$. The integrity of all plasmids was confirmed by DNA sequencing. All plasmids were purified using column purification (QIAGEN Inc., Valencia, California, USA) and each plasmid was prepared on at least two separate occasions.

$293 \mathrm{~T}$ cells were transfected in six-well plates so that that each well received $1.7 \mu \mathrm{g}$ of reporter, $0.9 \mu \mathrm{g}$ of either the MC4R or ObRb, $0.9 \mu \mathrm{g}$ of Stat isoform, or empty expression vector. When TR expression vectors were used, $0.9 \mu \mathrm{g}$ per well was used. Each well also received $20 \mathrm{ng}$ of a $\beta$-galactosidase expression vector. All transfections were performed using calcium phosphate as described previously (15). Sixteen hours after transfection, the cells were washed in PBS and refed. Twenty-eight hours after transfection, the cells were refed with serum-free Optimem (GIBCO BRL, Gaithersburg, Maryland, USA) and subsequently stimulated 48 hours after transfection with the indicated concentrations of leptin, $\alpha$-MSH, and T3. Eight hours after stimulation the cells were lysed and assayed for luciferase and $\beta$-galactosidase activity. The data shown is the mean of at least three separate experiments performed in triplicate.

Western analysis and electrophoretic mobility shift assays. To analyze phosphorylation of CREB by $\alpha-M S H, 293 \mathrm{~T}$ cells were stimulated for 15 minutes by $100 \mathrm{nM} \alpha-\mathrm{MSH}$. Nuclear extracts were isolated following the procedure of Schreiber et al. (25), and equal amounts were loaded onto a $10 \%$ SDS-PAGE. After electrophoresis, the proteins were transferred to nitrocellulose and probed overnight at $4^{\circ} \mathrm{C}$ with a $1: 1,000$ dilution of $\alpha$ CREB or $\alpha$-phospho-CREB antiserum (New England Biolabs Inc., Beverly, Massachusetts, USA). Western analysis was performed by ECL (Amersham Pharmacia Biotech, Piscataway, New Jersey, USA).
Electrophoretic mobility shift assays (EMSAs) were performed as described previously (26). Radiolabeled probes representing (a) Site 4 ( -83 to -36 of the hTRH promoter), (b) the putative Stat-responsive region of the human TRH promoter, (c) the analogous region of the murine TRH promoter ( -150 to -127 ; see Figure $5 \mathrm{~b})$, and (d) a canonical Stat-response element derived from the SIE sequence of the c-Fos promoter (SIEm67) (27) were incubated with the indicated nuclear extracts. Supershifts were carried out with the indicated antibodies ( $\alpha$-Flag, Sigma Chemical Co.) for an additional 20 minutes. Complexes were resolved on $5 \%$ nondenaturing polyacrylamide gels and visualized after autoradiography. EMSAs containing transfected Stats were only exposed for 2-4 hours, whereas all others were exposed overnight.

GST-protein interaction assays. The full-length cDNAs of the hTR $\beta$ isoforms were cloned into pGEX4T1 to yield GST fusion proteins in BL21 Escherichia coli. The GSTfusion proteins were incubated with $\left[{ }^{35} S\right]$ methionelabeled hSRC-1 or hCREB produced by in vitro translation. Interactions were performed as described previously (26). The concentration of T3 used was $1 \mu \mathrm{M}$.

\section{Results}

The melanocortin and leptin signaling pathways converge in the $P V H$. To determine whether leptin and/or the $\alpha-\mathrm{MSH}$ pathways were important in regulating TRH gene expression in the PVH, we performed double label in situ hybridization studies in Sprague-Dawley rats. As is shown in Figure 1, a and b, MC4R mRNA is expressed in

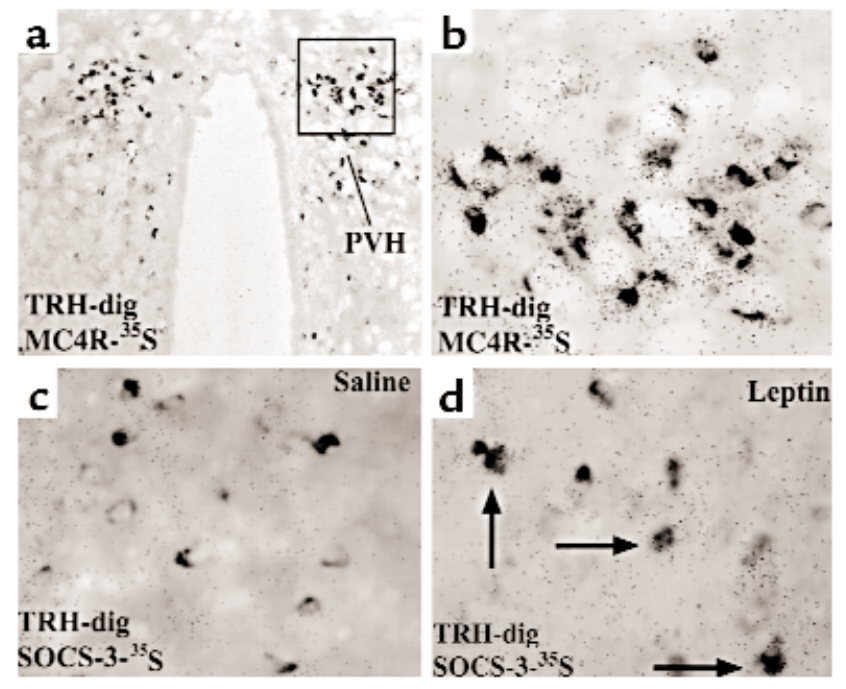

\section{Figure 1}

TRH neurons in the rat PVH express the MC4R and SOCS-3 after leptin administration. ( $\mathbf{a}$ and $\mathbf{b}$ ) Double label in situ hybridization was performed for the MC4R and TRH on hypothalamic sections. Shown is a caudal medial parvocellular section. TRH neurons are darkly stained (TRH-dig), whereas the granules represent MC4R expression (MC4R-35S). In this image, all the TRH neurons coexpress MC4R. (c and d) A similar study was performed to colocalize SOCS-3 (SOCS$3-{ }^{35 S}$ ) expression after saline or leptin treatment in rats fasted for 48 hours. The arrows indicate the double-labeled neurons. 


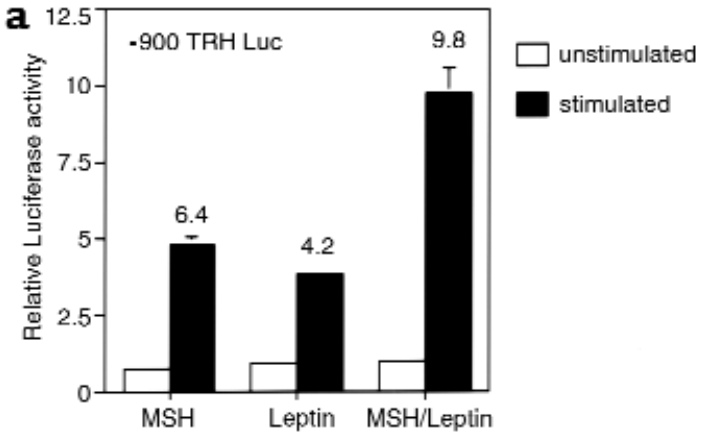

b

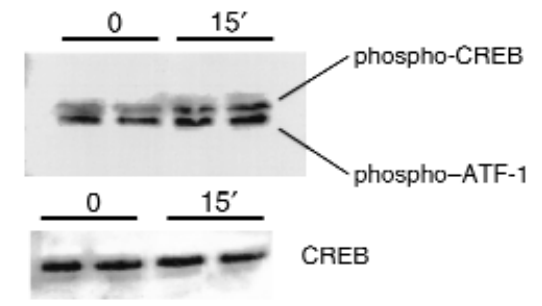

C

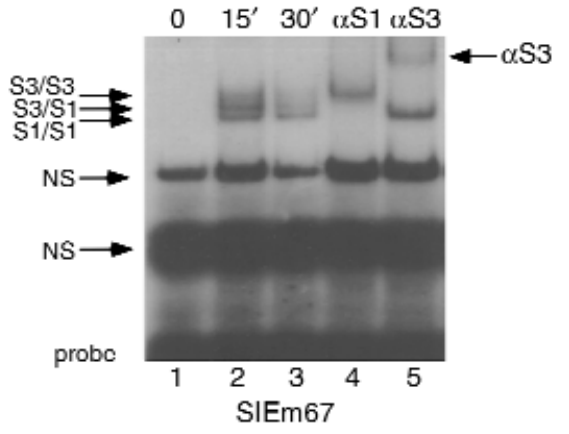

\section{Figure 2}

Leptin and $\alpha-\mathrm{MSH}$ signaling pathways activate the hTRH promoter. (a) 293T cells were transfected with both the MC4R and ObRb and a hTRH luciferase construct. Cells were stimulated with $100 \mathrm{nM}$ of the indicated ligand. The fold stimulation is shown over each treatment group. (b) Nuclear extract from 293T cells transfected in duplicate was prepared 15 minutes after stimulation with $\alpha-\mathrm{MSH}$. Equal amounts of protein from control and treated cells were probed using phosphoCREB and CREB antibodies. The duplicate results are shown. (c) Nuclear extracts were prepared from transfected 293T cells treated with leptin at the indicated times and used in EMSA with the radiolabeled SIEm67 element. Complexes were identified by using specific antibodies against Stat isoforms ( $\alpha \mathrm{S} 1$, against Stat $1 ; \alpha \mathrm{S} 3$, against Stat3). The antibodies were used on extract stimulated for 15 minutes.

the $\mathrm{PVH}$, suggesting the potential for $\alpha-\mathrm{MSH}$ signaling. Moreover, TRH neurons in the PVH coexpress MC4R mRNA, consistent with the hypothesis that the leptininduced production of $\alpha-\mathrm{MSH}$ in the arcuate nucleus engages the TRH-producing neurons in the PVH. We found that in the medial parvocellular divisions of the $\mathrm{PVH}, 48 \%$ of TRH neurons also expressed the MC4R.

To assess the role of leptin in the PVH, we assessed SOCS-3 mRNA expression in rats that had been fasted for 48 hours. We used the identical probe for TRH and examined for the expression of SOCS-3 mRNA in rats treated with saline or leptin (Figure 1, c and d). We found that SOCS-3 mRNA expression is induced in leptin-responsive neurons of the PVH. Expectedly, we also observed dense hybridization in the arcuate nucleus $(17,19)$. In saline-treated rats SOCS-3 expression was not seen in the arcuate or PVH (Figure 1c). In the leptin-treated rats (Figure 1d), SOCS-3 mRNA is coexpressed in a subset of TRH-positive neurons. Approximately $10 \%$ of TRH neurons are positive for SOCS- 3 in the medial parvocellular divisions of the PVH. Taken together, these data demonstrate that in fasted animals, leptin has the ability both to directly and to indirectly regulate TRH gene expression in the $\mathrm{PVH}$.

The TRH promoter is induced by both leptin and $\alpha-M S H$ in a heterologous system. To further explore the molecular basis for leptin and $\alpha-\mathrm{MSH}$ regulation of TRH gene expression, we developed a heterologous system, as hypothalamic cell lines to analyze directly TRH gene expression are not available. In this system, the mammalian 293T-cell line is cotransfected with the MC4R or ObRb and the human TRH promoter $(-900$ to +55$)$ linked to the luciferase reporter to assess the ability of these pathways to regulate TRH gene expression. As shown in Figure 2a, in the presence of $100 \mathrm{nM}$ of their respective ligands, both signaling pathways induce significant activation of the TRH promoter. In contrast, a control promoter, RSV180, was unaffected by both the MC4R and ObRb pathways (data not shown), indicating that the hTRH promoter is a specific target for both the MC4R and ObRb pathways. Furthermore, when treated with both ligands, the stimulation of the TRH promoter is additive, implying that leptin and $\alpha$-MSH act through separate pathways.

Given that leptin signaling is known to be mediated principally through the activation of Stat 3 and that the MC4R signals through the protein kinase A/CREB pathway, we next asked whether these individual pathways could be activated directly in the transfected cells. Figure $2 \mathrm{~b}$ reveals that cells transfected with the MC4R and stimulated with $\alpha-\mathrm{MSH}$ show an increase in phosphorylated CREB (P-CREB) compared with unstimulated cells. This demonstrates specific activation of the CREB pathway by MC4R signaling. The phosphoCREB antibody also recognizes other members of the activated transcription factor family (ATF).

To examine Stat activation by the ObRb, we performed EMSA with nuclear extracts derived from 293T cells transfected with the ObRb and either stimulated or not stimulated with $100 \mathrm{nM}$ leptin. As is shown in Figure 2c, leptin stimulation leads to the appearance of three specific bands that bind to the canonical Statresponsive element (SIEm67) used (lane 2). These bands are likely to represent the endogenous Stat 1 homodimer (lower band), the endogenous Stat3 homodimer (upper band), and a Stat1/Stat3 heterodimer (middle band). The use of specific antibodies demonstrates this, as the Stat 1 antibody blocks all endogenous Stat 1 binding (Figure 2c, lane 4), leaving only Stat3 homodimers to bind, whereas the Stat 3 antibody supershifts all Stat3 (Figure 2c, lane 5), leaving Stat 1 homodimers to bind. Thus, the ObRb pathway is able to activate and phos- 

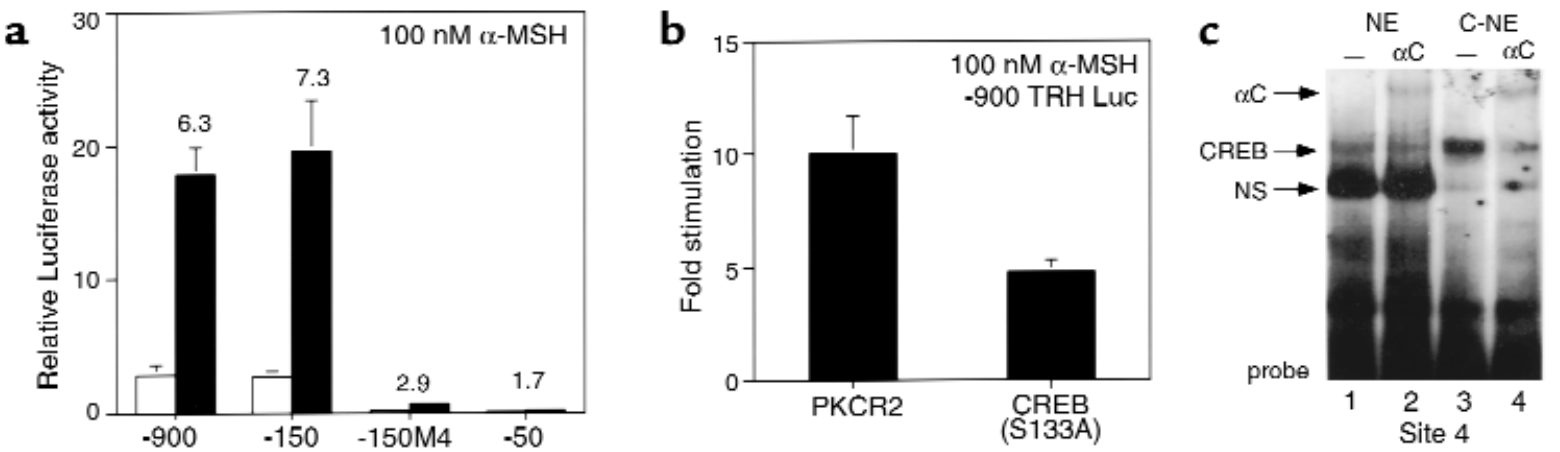

Figure 3

A near consensus CAMP response element mediates the $\alpha-M S H$ response via CREB. (a) The cis-acting sequence mediating the response to $\alpha$-MSH was mapped in 293T cells using the indicated hTRH promoter complexes. (b) A dominant CREB mutant (S133A) or empty vector (PKCR2) was cotransfected in the presence of the $-900 \mathrm{TRH}$ reporter construct, and the effects on stimulation were examined after the addition of $\alpha-\mathrm{MSH}$. The data are expressed as fold stimulation. (c) The binding of CREB to Site 4 was assessed using EMSA. Control (NE) or CREB-transfected (C-NE) nuclear extract was incubated with a radiolabeled Site 4 probe. The specificity of CREB binding is demonstrated by its supershift with anti-CREB $(\alpha C)$ antiserum.

phorylate Stat 3 as well as Stat 1 in 293 T cells. These data are consistent with those of others who have demonstrated that the ObRb pathway can activate a number of the Stat isoforms; although in vivo, only Stat3 appears relevant to leptin signaling (28).

$A$ CRE mediates regulation of the $h T R H$ promoter by $\alpha-M S H$. Inspection of the human, rat, and murine TRH promoters demonstrates the presence of a conserved element that bears close homology to a canonical CRE (TGACGTCA). This element, TGACCTCA, located between -60 and -55 of the hTRH promoter, has been previously termed Site 4 by our laboratory and functions both as an important site of TR binding and for negative regulation of the hTRH promoter by T3. On the basis of its homology to a CRE, we hypothesized that it would mediate the effects of $\alpha$-MSH signaling through MC4Rs. To confirm this, we studied a number of TRH promoter deletion constructs in $293 \mathrm{~T}$ cells that were also cotransfected with the MC4R. As is shown in Figure 3a, stimulation with $\alpha$-MSH leads to a 6.3 - and 7.3-fold stimulation of the -900 and -150 hTRH promoter constructs, respectively. However, deletion to -50 , which removes Site 4 , causes a loss of most stimulation (1.7-fold) by $\alpha$-MSH and lowers basal activity. The importance of Site 4 is best demonstrated by the -150M4 construct, which contains a mutated Site 4 (TAAAATCA). This construct loses both basal activity and the ability to be stimulated by $\alpha$-MSH (2.7-fold). Thus, Site 4 mediates the principal effects of $\alpha-\mathrm{MSH}$ on the TRH promoter. To demonstrate the role of CREB in this pathway, we initially cotransfected a dominant inhibitor of CREB (S133A), which cannot be phospho-
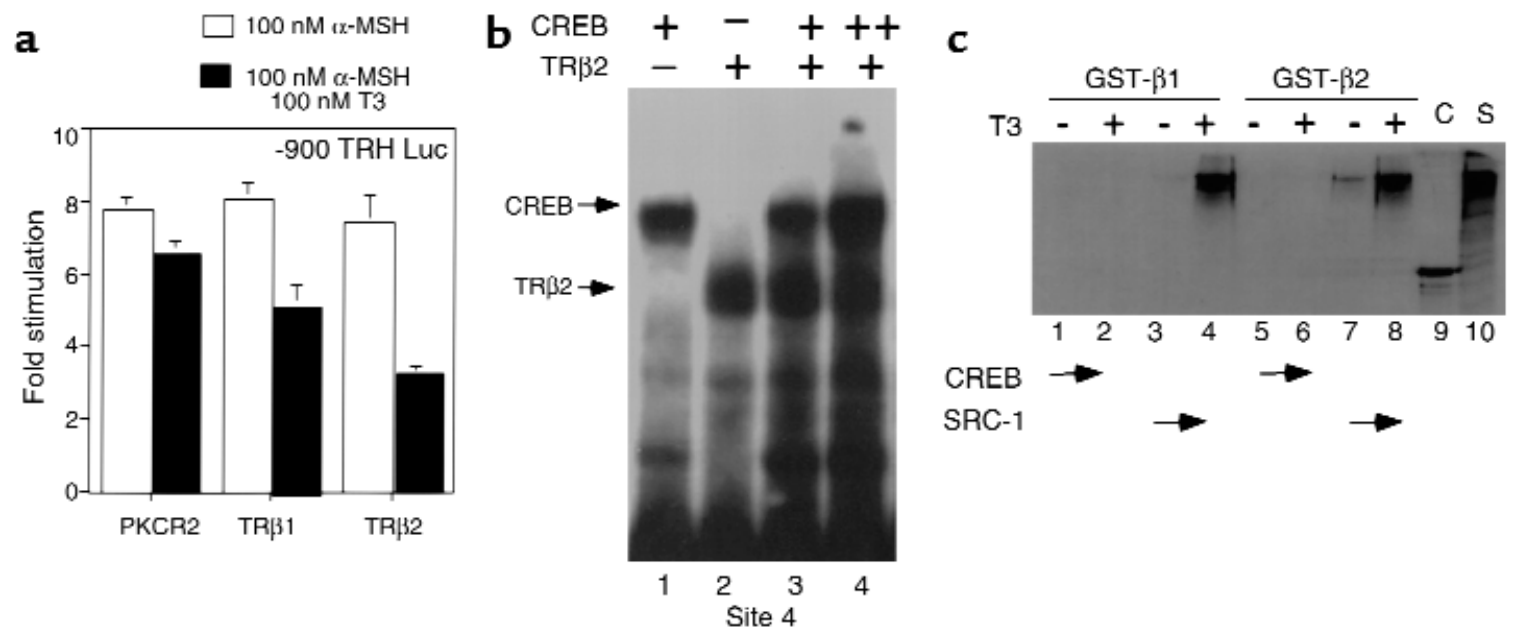

Figure 4

T3 blocks $\alpha$-MSH-mediated upregulation of the hTRH promoter. (a) 293T cells were cotransfected with expression vectors for the TR $\beta$ isoforms. The cells were incubated with either $100 \mathrm{nM} \alpha-\mathrm{MSH}$ alone or with $\alpha-\mathrm{MSH}$ and $100 \mathrm{nMT3}$. The data are expressed as fold stimulation from untreated cells. (b) EMSA was performed with radiolabeled Site 4 and CREB-transfected nuclear extract in the presence or absence of in vitro translated hTR $\beta 2$. (c) GST-TR $\beta 1$ or GST-TR $\beta 2$ was incubated with radiolabeled SRC-1 or CREB either in the presence or absence of 1 $\mu \mathrm{M}$ T3. Lane 9, CREB (C) input. Lane 10, SRC-1 (S) input. 


\section{Figure 5}

Leptin responsiveness maps to a specific region of the hTRH promoter. (a) 293T cells were transfected with a variety of hTRH reporter constructs. Their response to $100 \mathrm{nM}$ leptin was then determined. The data are expressed as relative luciferase activity, and the fold response is indicated above each construct. (b) The promoter sequences of the human, rat, and murine TRH genes are compared in the area of Stat responsiveness. The Stat-responsive element is boldface and located between -141 and -132 . The SP-1 site is boldface and underlined and located between -125 and -119 . Also shown are the mutations made to create the Statm and $\triangle \mathrm{SP}-1$ constructs. (c) 293T cells were transfected with the mTRH reporter construct in an identical paradigm, and its response to leptin was assessed. nTRE, negative thyroid hormone response element.

rylated by PKA in an identical paradigm. As indicated in Figure 3b, CREB S133A is able to block TRH promoter stimulation by $\alpha$-MSH by more than $50 \%$, consistent with the hypothesized role of CREB in mediating the effect of $\alpha-\mathrm{MSH}$. Finally, to demonstrate that CREB can indeed bind to Site 4, we performed EMSA with nuclear extract from both nontransfected and CREB-transfected 293T cells and can demonstrate (Figure 3c) that both endogenous CREB (lane 1) and transfected CREB (lane 3) bind strongly to the radiolabeled Site 4 probe and are specifically supershifted by CREB antiserum (lanes 2 and 4). Interestingly, the diminution in the nonspecific band (compare Figure 3c, lanes 1 and 2 to Figure $3 c$, lanes 3 and 4 ) in the presence of transfected CREB suggests that other nuclear complexes interact with Site 4 . Taken together, these data demonstrate that $\alpha-\mathrm{MSH}$ stimulates the hTRH promoter through the CREB signaling pathway via a direct interaction with a near canonical CRE in the proximal portion of the promoter. This is likely to be an important pathway for TRH regulation in all species given that Site 4 is conserved in the TRH promoter of all species studied to date. Furthermore, CREB is an important transcriptional regulator of other $\mathrm{PVH}$ expressed genes including corticotropin-releasing hormone (CRH) and vasopressin (29-31).

Given the role of Site 4 in mediating $\alpha$-MSH-mediated stimulation and its previously described role in binding the TR (15), we next asked whether T3 could inhibit the upregulation of the TRH promoter by $\alpha$ MSH. To do this, we cotransfected either empty expression vector or the human TR $\beta 1$ or TR $\beta 2$ isoforms. In transfection experiments, the TR $\beta 1$ isoform is a more potent activator of transcription on positive thyroid hormone response elements in $293 \mathrm{~T}$ cells (data not shown). In the presence of $\alpha-\mathrm{MSH}, \mathrm{T} 3 \mathrm{had}$ minimal effect unless cotransfected TR was present (Figure 4a). Cotransfected TR $\beta 1$ in the presence of T3 had some ability to block $\alpha$-MSH stimulation of the TRH promoter, but TR $\beta 2$ inhibited stimulation by over $50 \%$. These data are consistent with the increased ability of TR $\beta 2$ to mediate negative regulation both in vitro and in vivo $(32,33)$. Although both the TR and CREB can bind to Site 4 (Figure $4 \mathrm{~b}$ ), there is no evidence that an

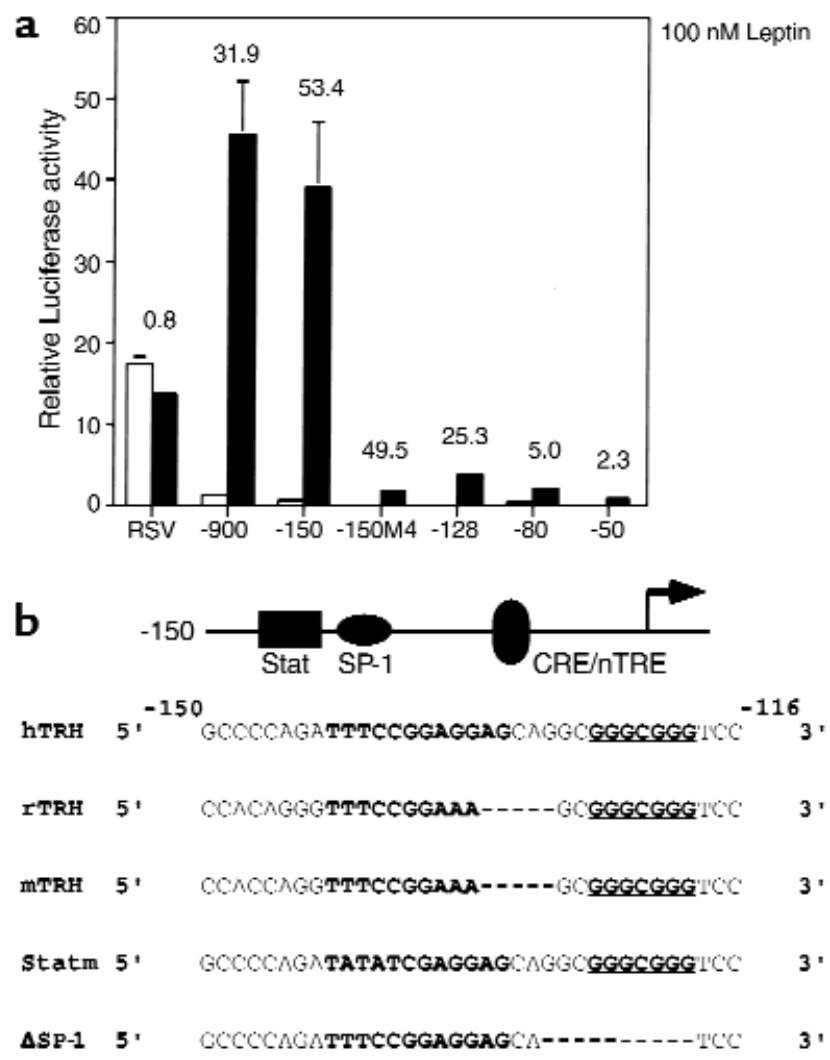

C

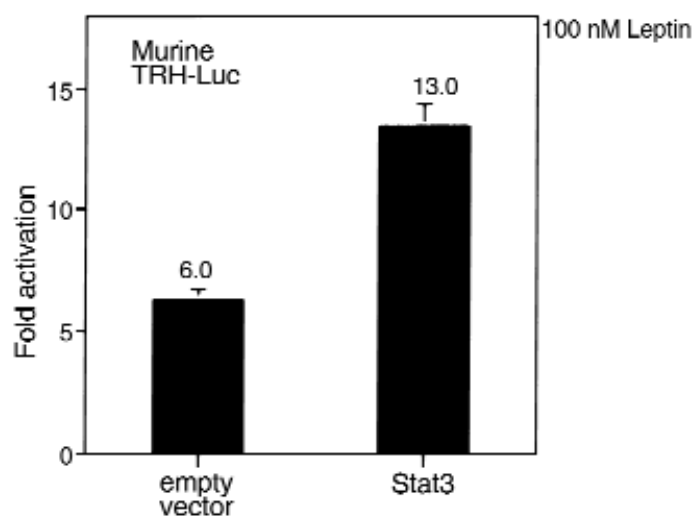

interaction between the two occurs using EMSA, even in the presence of ligand (data not shown). To investigate further whether the TR isoforms could interact with CREB, we used a GST pull-down assay in which each of the TR isoforms were produced as GST fusions. As shown in Figure 4c, TR $\beta 1$ can recruit the nuclear receptor coactivator SRC-1 in the presence of T3 only, whereas, as we have shown previously, $\mathrm{TR} \beta 2$ can recruit SRC- 1 in both the presence and absence of T3 (34). In contrast, neither could recruit CREB in the presence or absence of ligand. Further study will be required to define the mechanisms by which $\mathrm{T} 3$ inhibition occurs.

The hTRH promoter is regulated by leptin in a Stat3-specific fashion. To identify the region of the hTRH promoter necessary for regulation by leptin through the ObRb, we again used a variety of hTRH promoter constructs. Because of the relative paucity of Stat 3 in 293T cells 
(35), we also cotransfected a Stat3 expression vector in our initial mapping studies. As shown in Figure 5a, the full-length human TRH promoter is stimulated 31.9fold by $100 \mathrm{nM}$ leptin in the presence of cotransfected $\mathrm{ObRB}$ and Stat3. A dose-response experiment was also performed, and similar effects were seen with $1 \mathrm{nM}$ and $10 \mathrm{nM}$ leptin (data not shown). The control RSV Luc construct is not activated at all in this paradigm. These data support the notion that direct signaling by the $\mathrm{ObRb}$ on the TRH promoter is mediated by the JAKStat pathway. We then used a variety of deletion constructs to map the Stat-responsive region of the promoter. Deletion of the promoter to -150 (Figure 5a) does not alter the response (53.4-fold). However, deletion to -128 causes a $50 \%$ reduction in response, whereas deletion to -80 and -50 causes the response to be virtually lost. Mutation of the CREB binding site $(-150 \mathrm{M} 4)$ does not affect the responsiveness of the promoter (49.5-fold) to the Stat pathway, but again decreases basal activity. Thus, the cis-acting sequences mediating Stat 3 responsiveness of the TRH promoter lie between -150 and -80 , with key sequences located between -150 and -128 .

We next analyzed the human TRH promoter sequence and those of the rat and mouse in the areas of relevance. Indeed, a site resembling a Stat-response element (TTCCGGAGGA; Figure 5b) is present between -141 and -132 of the human TRH promoter. Furthermore, a canonical Stat-response element (TTCCGGAAAG) is present at the analogous location in the murine and rat promoters. In addition to the Stat element, both the human and rodent promoters have a highly conserved GC box just downstream from the putative Stat element that we have shown can bind SP-1 (underlined and bold in Figure 5b; and data not shown). This is significant given the known importance of SP-1 in helping to mediate the Stat response and potentially to interact with a number of the Stat isoforms in context of other promoters $(36,37)$. To confirm that the rodent promoter could indeed respond to the leptin signaling pathway, we used an identical paradigm with a murine TRH promoter construct $(-255$ to +5$)$ that contains the Stat binding element. Like its human counterpart, it was stimulated well by leptin in the presence of ObRb (sixfold), and this response was enhanced by the presence of cotransfected Stat3 (13-fold; Figure 5c).

Given the presence of a putative Stat binding site and SP-1 site in the human and rodent TRH promoters, we next assessed the import of each of these sites by either mutating or deleting them in context of the -150 human construct. As is shown in Figure 6a, mutagenesis of the Stat-responsive element (Statm) causes a more than $60 \%$ drop in responsiveness to leptin. Similarly, deletion of the Stat binding site $(-128)$ also causes a similar drop in the responsiveness to leptin. Deletion of the SP-1 site $(\Delta$ SP- 1$)$ only causes a $20 \%$ loss of leptin responsiveness. As was demonstrated previously, deletion of both sites ( -80 construct) causes a complete loss of response to leptin. Taken together, the transfection data support the notion that Stat 3 and SP-1 cooperate to mediate the leptin response. However, the Stat 3 site alone is more powerful. To determine whether the Statresponsive region could indeed bind Stat3, we performed EMSA with the radiolabeled human element. Nuclear extracts from $293 \mathrm{~T}$ cells cotransfected with $\mathrm{ObRb}$ and Stat 3 were prepared both before and $15 \mathrm{~min}$ utes after stimulation with $100 \mathrm{nM}$ leptin. Stat 3 binds to the human probe and is supershifted with the Flag antibody (Figure 6b). However, this binding was not nearly as strong as that seen with the idealized Stat
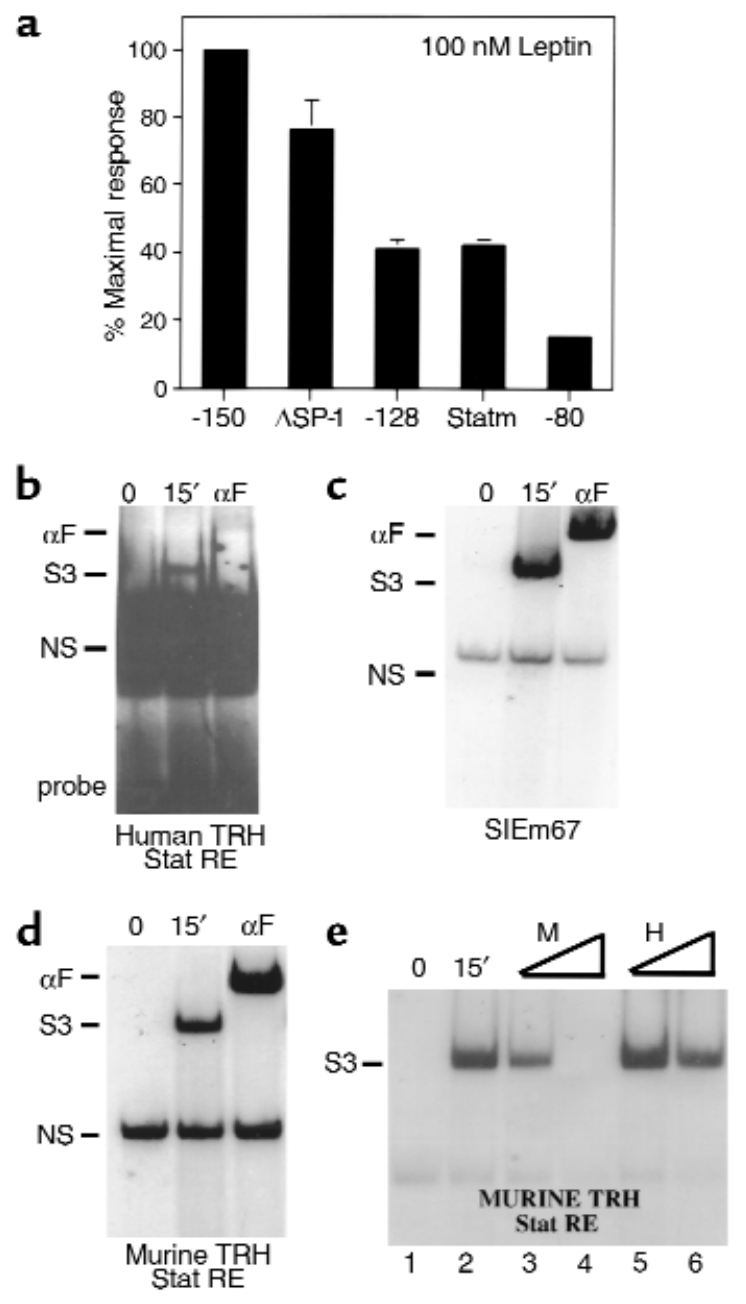

\section{Figure 6}

Identification of the Stat binding site in the TRH promoter. (a) 293T cells were cotransfected with the indicated hTRH constructs. Leptin responsiveness was then determined. The data are expressed as percent of maximal response where the fold stimulation of the -150 construct is set at $100 \%$. (b) EMSA was performed with a radiolabeled probe spanning the human Stat site and nuclear extract from leptintreated or untreated cells. The specificity of the bound complex was discerned by using an anti-Flag ( $\alpha F)$ antibody. (c) A similar EMSA paradigm was used on the canonical SIEm67 probe. (d) A similar EMSA paradigm was used on a probe spanning the murine Stat element. (d) EMSA was performed on the murine Stat element with the addition of cold oligonucleotides representing the murine (lanes 3 and 4) or human (lanes 5 and 6) Stat elements. A $10 \times$ cold competitor was added in lanes 3 and 5, and 100x in lanes 4 and 6 . 


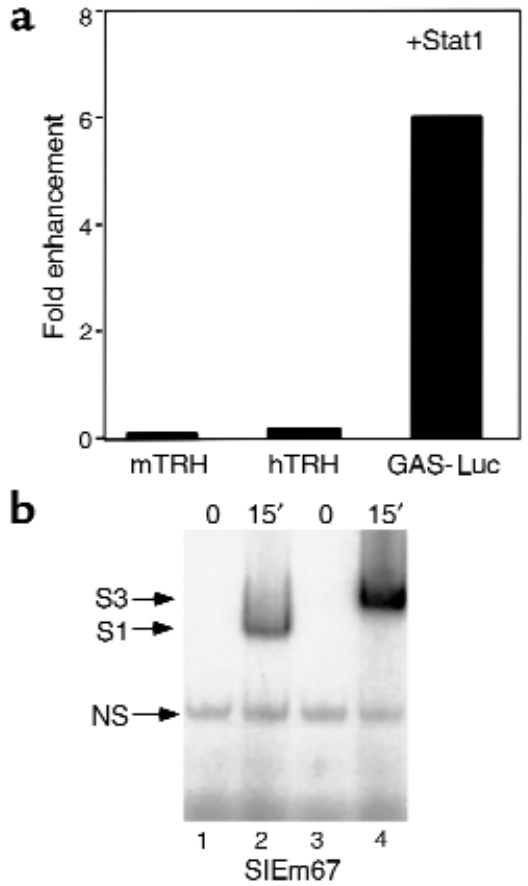

\section{Figure 7}

The effect of Stat isoforms on TRH promoter activity. (a) 293T cells were cotransfected with the indicated TRH constructs or Gas-Luc with ObRb in the presence or absence of Stat 1 . The effect of Stat 1 is quantified as fold enhancement over values from cells that did not receive Stat1. (b) EMSA was performed with the canonical SIEm67 probe and NE from Stat 1 or Stat 3 transfected cells. The indicated complexes are shown.

SIEm67 element (Figure 6c). To compare Stat binding on the human site versus that found with murine element, we again performed EMSA, this time using the murine TRH Stat-response element as a probe. As expected, given its canonical sequence, the murine response element binds Stat 3 with an avidity near that of the idealized element (Figure 6d). In addition, the entire complex is supershifted with an anti-flag antibody. To compare avidity for Stat 3 binding, we performed competition studies using both excess cold human and murine probe with radiolabeled murine probe. These studies reveal (Figure 6e) that the human site can compete with the murine site when present in 100 -fold excess. However, 100 -fold excess human probe competes in a weaker fashion than does tenfold excess cold murine probe (compare lane 3 to lane 6 in Figure $6 \mathrm{e})$, whereas 100 -fold excess cold murine probe competes completely for binding (Figure 6e, lane 4). Thus, the human site binds Stat 3 with an avidity of at least 15to 20 -fold less than the analogous rodent site. It should be pointed out that an analogous noncanonical Stat3 element in the murine C/EBP $\delta$ promoter binds Stat3 very weakly, if at all, in EMSA and also requires SP-1 for full transcriptional activity (37).

Data from two separate groups have shown that leptin specifically activates the Stat 3 isoform in the hypothalamus $(38,39)$. To determine whether the activation of the TRH promoters was Stat isoform specific, we examined the ability of Stat 1 to activate the human or murine TRH promoters. As a control, we used a multimerized Stat-response element from the IFN- $\gamma$ promoter upstream of luciferase (GAS-Luc). Stat 1 was unable to increase activation of the hTRH promoter or the $\mathrm{mTRH}$ promoter. However, Stat 1 is able to activate the response of the GAS-Luc construct by sixfold over levels seen in cells without transfected Stat1 (Figure $7 \mathrm{a}$ ). These data are consistent with Stat 1 being phosphorylated by the leptin pathway in 293T cells (Figure $7 \mathrm{~b})$ but suggest that the TRH promoter responds preferentially to Stat3.

\section{Discussion}

Central to the actions of leptin are its ability to regulate hypothalamic expression of neuropeptide genes, which in turn control food intake and energy expenditure. However, the molecular mechanisms governing the regulation of target genes by leptin are not well understood. Recently, it has become clear that leptin is able to regulate thyroid hormone production in rodents in situations in which the conservation of energy through a reduction in metabolic rate is necessary $(4,40)$. The regulation of the thyroid hormone axis by leptin appears to occur principally at the level of the TRH neuron in the PVH. In this model, leptin could either act directly through receptors on the TRH neuron or indirectly, via other hypothalamic neurons that project to the PVH (reviewed in ref. 41). It is established that production of $\alpha-\mathrm{MSH}$ in the arcuate nucleus, which is increased by leptin, increases TRH gene expression and increases TRH synthesis in the hypothalamus $(9,10)$. Whether this effect is mediated by melanocortin signaling on these TRH neurons has not been addressed. The data presented here demonstrate the coexpression of MC4R by TRH neurons in the $\mathrm{PVH}$, confirming that the melanocortin system is poised to regulate TRH gene expression by direct action on these cells.

Direct signaling by leptin to the TRH neuron has been more difficult to discern. However, previous work has demonstrated the existence of the ObRb in the $\mathrm{PVH}$ and that leptin can induce c-Fos expression in the PVH $(13,14)$. In addition, leptin administration induces the expression of SOCS-3 mRNA in a circumscribed population of PVH neurons (19). Furthermore, in cultured primary rat fetal hypothalamic neurons, the ObRB and TRH colocalize to single neurons using immunocytochemistry (12). Here, we demonstrate the coexpression of SOCS-3 and TRH message in a subset of PVH neurons in fasted rats stimulated with leptin. Because SOCS-3 is a sensitive marker of direct leptin action, its presence in even a small percentage of TRH neurons implies that leptin can directly act on the TRH neuron, although the anatomical data suggest that the melanocortin pathway may play a larger role.

To examine the molecular basis by which the MC4 and leptin receptor signaling pathways induce TRH gene expression, we developed a transfection system in 
heterologous mammalian cells. Our data demonstrates that the MC4R and $\mathrm{ObRb}$ signaling pathways are able specifically to activate the human TRH promoter through distinct cis-acting elements. The MC4R, like the other members of the melanocortin receptor family signals by increasing levels of intracellular cAMP (42), which in turn leads to the phosphorylation of CREB, which activates transcription through the recruitment of the coactivator CREB-binding protein (CBP) (43). We have clearly demonstrated that CREB can be phosphorylated through MC4R activation in $293 \mathrm{~T}$ cells and that CREB binds strongly to a conserved region in the human and rodent TRH promoter (Site 4). The MC4R signaling pathway activates the TRH promoter through Site 4 and cotransfection of a CREB mutant that cannot be phosphorylated inhibits MC4R-mediated activation by more than $50 \%$. Thus, the MC4R pathway specifically activates the TRH promoter through the phosphorylation and binding of CREB. Given the hypothalamic expression of CREB (data not shown and ref. 44), this mechanism is likely to be operative in vivo and further strengthens the link between the regulation of TRH synthesis and secretion by $\alpha-M S H$.

Interestingly, CREB-mediated stimulation of the TRH promoter is inhibited by the TR $\beta 2$ isoform in the presence of T3. This is consistent with in vivo data demonstrating that the TRH gene is downregulated by $\mathrm{T} 3$ and by more recent data demonstrating that the $\mathrm{TR} \beta 2$ isoform is necessary for negative regulation by T3 (33). Further work will be required to discern the relative expression of these transcription factors and their relative cofactors in the $\mathrm{PVH}$, which should provide further insight into the mechanisms governing negative regulation by $\mathrm{T} 3$.

In addition to the MC4R, the ObRb signaling pathway stimulates the expression of the TRH gene directly. It is clear from our data that this stimulation is Stat dependent and not mediated by the CREB/ATF pathway. Our mapping experiments have localized the main Stat-responsive region to the nucleotides between -150 and -125 of the hTRH promoter. Analysis of this region across species demonstrates the presence of a canonical Stat-response element in the murine and rat TRH promoters that binds Stats avidly (Figure 6). Interestingly, this site has changed slightly in the human promoter, but still convincingly mediates Stat responsiveness. However, it does interact much more weakly with Stat3. This divergence in the Stat-response element may enable Stat isoform specificity in vivo but this remains to be shown. The availability of specific inhibitors of Stat isoforms, the protein inhibitors of activated Stat 1 and 3 (45), should allow us to explore isoform specificity further in the context of human and rodent promoters.

Analysis of other Stat-responsive genes suggests that the hTRH promoter is similar in structure to the murine C/EBP $\delta$ promoter that is also responsive only to Stat3 (37). This promoter, like the human and rodent TRH promoter, also contains an SP-1 site that is located next to the Stat-responsive element. This is necessary for full induction of the murine $\mathrm{C} / \mathrm{EBP} \delta$ promoter by Stat 3 . In addition, like the hTRH promoter, the murine $\mathrm{C} / \mathrm{EBP} \delta$ promoter can partially respond to Stat 3 via its SP-1 site only. Similarly, the ICAM-1 gene requires both Stat and SP-1 sites in its proximal promoter in order to respond fully to IFN- $\gamma(36)$. Indeed Look et al. were able to show a physical interaction between Stat 1 and SP-1 (36). Taken together, these data demonstrate that the leptin response of the hTRH promoter is mediated, by Stat 3 in conjunction with SP- 1 . This pathway is preserved in the murine and rat TRH promoters, which validates their use as models to explore leptin signaling in vivo.

The data presented here importantly extend our understanding of the direct and indirect mechanisms by which leptin activates TRH gene expression in the PVH. The transcriptional pathways engaged by both MC4 and leptin receptor signaling are targeted through distinct transcription factor binding sites in the proximal TRH promoter and allow its positive regulation. Thus, thyroid hormone production can be controlled dynamically through the transcriptional regulation of the TRH promoter through the interplay of leptin, $\alpha-\mathrm{MSH}$ and T3. These pathways allow leptin to directly regulate energy expenditure and provide a link between food consumption and energy expenditure. At the molecular level, the TRH promoter emerges as the best-defined targets of leptin action on gene expression. Given the potential role of other hormonal pathways, such as NPY (46) in the $\mathrm{PVH}$, it is likely that other inputs to this promoter will also be important (Figure 8). The transcriptional regulation of TRH by pathways separate from T3 will also

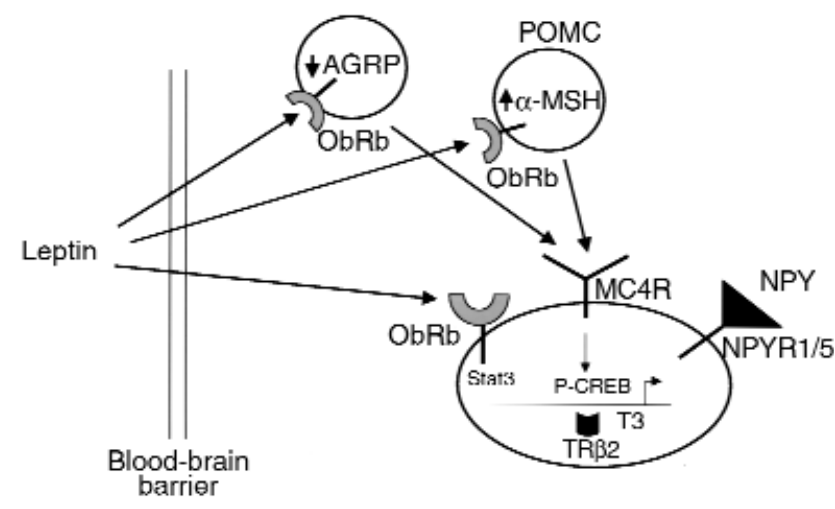

\section{Figure 8}

A model of leptin action on the TRH neuron. Shown is a proposed model of leptin action on the TRH neuron. After crossing the bloodbrain barrier leptin can act either directly on the TRH neuron or via an indirect pathway by increasing the production of $\alpha-\mathrm{MSH}$ in the POMC neuron in the arcuate nucleus. Also shown are the inputs of AGRP and NPY, both of which are known to project to the TRH neuron. Leptin, through the phosphorylation of Stat3, can directly activate the TRH promoter as can $\alpha-M S H$ through the activation of CREB. T3 can inhibit TRH gene expression, principally through the TR $\beta 2$ isoform. NPYR1/5, NPY receptor 1 or 5. 
likely be important in the adaptation of the thyroid hormone axis in other physiological situations besides starvation, such as severe illness, in which T3 levels and TSH levels can be suppressed (47).

\section{Acknowledgments}

This work was supported by grants from the NIH to J.S. Flier (DK 28082 and 46930), J.E.Elmquist (DK 56116 and DK 53301), and A.N. Hollenberg (DK 57658 and DK 56123). We thank I. Gantz, M. Yamada, and K. Shuai for plasmids, and Sabrina Brzostek for technical assistance.

1. Yamada, M., et al. 1997. Tertiary hypothyroidism and hyperglycemia in mice with targeted disruption of the thyrotropin-releasing hormone gene. Proc. Natl. Acad. Sci. USA. 94:10862-10867.

2. Segerson, T.P., et al. 1987. Thyroid hormone regulates TRH biosynthesis in the paraventricular nucleus of the rat hypothalamus. Science. 238:78-80.

3. Blake, N.G., Eckland, D.J., Foster, O.J., and Lightman, S.L. 1991. Inhibition of hypothalamic thyrotropin-releasing hormone messenger ribonucleic acid during food deprivation. Endocrinology. 129:2714-2718.

4. Ahima, R.S., et al. 1996. Role of leptin in the neuroendocrine response to fasting. Nature. 382:250-252.

5. Legradi, G., Emerson, C.H., Ahima, R.S., Flier, J.S., and Lechan, R.M. 1997. Leptin prevents fasting-induced suppression of prothyrotropinreleasing hormone messenger ribonucleic acid in neurons of the hypothalamic paraventricular nucleus. Endocrinology. 138:2569-2576.

6. Legradi, G., et al. 1998. Arcuate nucleus ablation prevents fastinginduced suppression of ProTRH mRNA in the hypothalamic paraventricular nucleus. Neuroendocrinology. 68:89-97.

7. Thornton, J.E., Cheung, C.C., Clifton, D.K., and Steiner, R.A. 1997. Regulation of hypothalamic proopiomelanocortin mRNA by leptin in ob/ob mice. Endocrinology. 138:5063-5066.

8. Mizuno, T.M., et al. 1998. Hypothalamic pro-opiomelanocortin mRNA is reduced by fasting and in ob/ob and $\mathrm{db} / \mathrm{db}$ mice, but is stimulated by leptin. Diabetes. 47:294-297.

9. Fekete, C., et al. 2000. alpha-Melanocyte-stimulating hormone is contained in nerve terminals innervating thyrotropin-releasing hormonesynthesizing neurons in the hypothalamic paraventricular nucleus and prevents fasting-induced suppression of prothyrotropin-releasing hormone gene expression. J. Neurosci. 20:1550-1558.

10. Kim, M.S., et al. 2000. The central melanocortin system affects the hypothalamo-pituitary thyroid axis and may mediate the effect of leptin. J. Clin. Invest. 105:1005-1011.

11. Ollmann, M.M., et al. 1997. Antagonism of central melanocortin receptors in vitro and in vivo by agouti-related protein [erratum 1998 , 281:1615). Science. 278:135-138.

12. Nillni, E.A., et al. 2000. Leptin regulates prothyrotropin-releasing hormone biosynthesis: evidence for direct and indirect pathways. J. Biol. Chem. 275:36124-36133.

13. Elmquist, J.K., Bjorbaek, C., Ahima, R.S., Flier, J.S., and Saper, C.B. 1998. Distributions of leptin receptor mRNA isoforms in the rat brain. $J$ Comp. Neurol. 395:535-547.

14. Elias, C.F., et al. 2000. Chemical characterization of leptin-activated neurons in the rat brain. J. Comp. Neurol. 423:261-281.

15. Hollenberg, A.N., et al. 1995. The human thyrotropin-releasing hormone gene is regulated by thyroid hormone through two distinct classes of negative thyroid hormone response elements. Mol. Endocrinol. 9:540-550.

16. Satoh, T., Yamada, M., Iwasaki, T., and Mori, M. 1996. Negative regulation of the gene for the preprothyrotropin-releasing hormone from the mouse by thyroid hormone requires additional factors in conjunction with thyroid hormone receptors. J. Biol. Chem. 271:27919-27926.

17. Elias, C.F., et al. 1998. Leptin activates hypothalamic CART neurons projecting to the spinal cord. Neuron. 21:1375-1385

18. Marcus, J.N., et al. 2001. Differential expression of orexin receptors 1 and 2 in the rat brain. J. Comp. Neurol. In press.

19. Bjorbaek, C., Elmquist, J.K., Frantz, J.D., Shoelson, S.E., and Flier, J.S. 1998. Identification of SOCS-3 as a potential mediator of central leptin resistance. Mol. Cell. 1:619-625.

20. Elmquist, J.K., Ahima, R.S., Maratos-Flier, E., Flier, J.S., and Saper, C.B. 1997. Leptin activates neurons in ventrobasal hypothalamus and brain- stem. Endocrinology. 138:839-842.

21. Marks, D.L., et al. 1992. Simultaneous visualization of two cellular mRNA species in individual neurons by use of a new double in situ hybridization method. Mol. Cell. Neurosci. 3:395-405.

22. Hollenberg, A.N., et al. 1997. Functional antagonism between CCAAT/Enhancer binding protein alpha and peroxisome proliferatoractivated receptor gamma on the leptin promoter. J. Biol. Chem. 272:5283-5290

23. Bjorbaek, C., Uotani, S., da Silva, B., and Flier, J.S. 1997. Divergent signaling capacities of the long and short isoforms of the leptin receptor. Biol. Chem. 272:32686-32695.

24. Hollenberg, A.N., Monden, T., and Wondisford, F.E. 1995. Ligand-independent and -dependent functions of thyroid hormone receptor isoforms depend upon their distinct amino termini. J. Biol. Chem. 270:14274-14280.

25. Schreiber, E., Matthias, P., Muller, M.M., and Schaffner, W. 1989. Rapid detection of octamer binding proteins with "mini extracts", prepared from a small number of cells. Nucleic Acids Res. 17:6419.

26. Monden, T., Wondisford, F.E., and Hollenberg, A.N. 1997. Isolation and characterization of a novel ligand-dependent thyroid hormone receptor-coactivating protein. J. Biol. Chem. 272:29834-29841.

27. Wagner, B.J., Hayes, T.E., Hoban, C.J., and Cochran, B.H. 1990. The SIF binding element confers sis/PDGF inducibility onto the c-fos promoter. EMBO J. 9:4477-4484.

28. Wang, Y., et al. 1997. Leptin receptor action in hepatic cells. J. Biol. Chem. 272:16216-16223.

29. Seasholtz, A.F., Thompson, R.C., and Douglas, J.O. 1988. Identification of cyclic adenosine monophosphate-responsive element in the rat corticotropin-releasing hormone gene. Mol. Endocrinol. 2:1311-1319.

30. Verbeeck, M.A., Adan, R.A., and Burbach, J.P. 1990. Vasopressin gene expression is stimulated by cyclic AMP in homologous and heterologous expression systems. FEBS Lett. 272:89-93.

31. Pardy, K., et al. 1992. The identification of a cis-acting element involved in cyclic 3',5'-adenosine monophosphate regulation of bovine vasopressin gene expression. J. Biol. Chem. 267:21746-21752.

32. Langlois, M.F., et al. 1997. A unique role of the beta-2 thyroid hormone receptor isoform in negative regulation by thyroid hormone. Mapping of a novel amino-terminal domain important for ligand-independent activation. J. Biol. Chem. 272:24927-24933.

33. Abel, E.D., et al. 1999. Divergent roles for thyroid hormone receptor beta isoforms in the endocrine axis and auditory system. J. Clin. Invest. 104:291-300.

34. Oberste-Berghaus, C., et al. 2000. Thyroid hormone-independent interaction between the thyroid hormone receptor beta2 amino terminus and coactivators. J. Biol. Chem. 275:1787-1792.

35. Bromberg, J.F., et al. 1999. Stat3 as an oncogene. Cell. 98:295-303

36. Look, D.C., Pelletier, M.R., Tidwell, R.M., Roswit, W.T., and Holtzman, M.J. 1995. Stat1 depends on transcriptional synergy with SP-1. J. Biol. Chem. 270:30264-30267.

37. Cantwell, C.A., Sterneck, E., and Johnson, P.F. 1998. Interleukin-6-specific activation of the $\mathrm{C} / \mathrm{EBP} \delta$ gene in hepatocytes is mediated by STAT3 and SP1. Mol. Cell. Biol. 18:2108-2117.

38. Vaisse, C., et al. 1996. Leptin activation of Stat3 in the hypothalamus of wild-type and ob/ob mice but not db/db mice. Nat. Genet. 14:95-97.

39. McCowen, K.C., Chow, J.C., and Smith, R.J. 1998. Leptin signaling in the hypothalamus of normal rats in vivo. Endocrinology. 139:4442-4447.

40. Flier, J.S. 1998. Clinical review 94: What's in a name? In search of leptin's physiologic role. J. Clin. Endocrinol. Metab. 83:1407-1413.

41. Flier, J.S., Harris, M., and Hollenberg, A.N. 2000. Leptin, nutrition, and the thyroid: the why, the wherefore, and the wiring. J. Clin. Invest. 105:859-861.

42. Yang, Y.K., et al. 1999. Characterization of Agouti-related protein binding to melanocortin receptors. Mol. Endocrinol. 13:148-155.

43. Chrivia, J.D., et al. 1993. Phosphorylated CREB binds specifically to the nuclear protein CBP. Nature. 365:855-859.

44. Kovacs, K.J., and Sawchenko, P.E. 1996. Sequence of stress-induced alterations in indices of synaptic and transcriptional activation in parvocellular neurosecretory neurons. J. Neurosci. 16:262-273.

45. Chung, C.D., et al. 1997. Specific inhibition of Stat 3 signal transduction by PIAS3. Science. 278:1803-1805.

46. Legradi, G., and Lechan, R.M. 1998. The arcuate nucleus is the major source for neuropeptide Y-innervation of thyrotropin-releasing hormone neurons in the hypothalamic paraventricular nucleus. Endocrinology. 139:3262-3270

47. Nicoloff, J.T., and LoPresti, J.S. 1996. Nonthyroidal illness. In The thyroid. L.E. Braverman and R.D. Utiger, editors. Lippincott-Raven. Philadelphia, Pennsylvania, USA. 286-296. 